\title{
Validation of a Liquid Chromatography/Tandem Mass Spectrometry Assay for the Quantification of Plasma Dihydroartemisinin
}

\author{
Dona Arlinda, Intan Sari Oktoberia, Muhammad Karyana \\ National Institute of Health Research and Development, Ministry of Health of Indonesia, Jakarta, Indonesia
}

Background: Insufficient plasma level of dihydroartemisinin (DHA) can select resistance and will further hinder malaria elimination program. We investigated clinical applicability of a validated liquid chromatography/tandem mass spectrometry (LC-MS/MS) assay to quantify plasma concentration of DHA in healthy subjects from a single oral administration of fixed dose combination of Dihydroartemisinin-Piperaquine.

Materials and Methods: Micro-elution solid-phase extraction in a 96-well plate format was used to prepare the samples. DHA separation happened in Acquity UPLC ${ }^{\mathrm{TM}}$ BEH C18 column $(50 \times 2.1 \mathrm{~mm}, 1.7 \mu \mathrm{m})$. Mobile phase was a mixture of acetonitrile-ammonium acetate $10 \mathrm{mM} \mathrm{pH} 3.5(50: 50, \mathrm{v} / \mathrm{v})$ at $0.3 \mathrm{~mL} /$ minute flow rate. Waters Acquity UPLC'M H-Class system coupled with triple quadruple mass spectrometry in positive electrospray ionization mode was used for detection. The internal standard was a stable isotope labelled DHA.

Results: Calibration curve was linear with a correlation coefficient $>0.995$ over a concentration range of $1-1,000 \mathrm{ng} / \mathrm{mL}$. Bias and variation for accuracy and precision were in the range of $15 \%$ (20\% at the lower limit of quantification). Using 5 $\mu \mathrm{L}$ sample, lower limit of quantification was $1 \mathrm{ng}$. Matrix effect was less than $15 \%$. The method was successfully applied to investigate the pharmacokinetics of DHA from five healthy subjects, although carry over and the role of anticoagulants were not tested.

Conclusion: The LC-MS/MS assay for the quantification of plasma DHA was validated for selectivity, linearity, lower limit of quantitation, accuracy, precision, matrix effect and stability. Although clinical applicability was demonstrated, this method was to be improved to address the not-tested validation parameters.

Keywords: dihydroartemisinin, liquid chromatography/tandem mass spectrometry assay (LC-MS/MS), malaria, Indonesia

\section{Introduction}

Artemisinin-based Combination Therapy (ACT) is a WHO-recommended treatment for uncomplicated malaria.
Dihydroartemisinin (DHA) together with piperaquine have been used as the first line ACT in Indonesia since $2008{ }^{1,2}$ Dihydroartemisinin is a reduced lactol derivative of artemisinin with a common pharmacopore of 1,2,4-trioxane.

Date of submission: October 29, 2020

Last Revised: September 20, 2021

Accepted for publication: September 21, 2021

Corresponding Author:

Dona Arlinda

National Institute of Health Research and Development

Ministry of Health of Indonesia

Jl. Percetakan Negara No.29, Jakarta 10560, Indonesia

e-mail: arlindona1@gmail.com

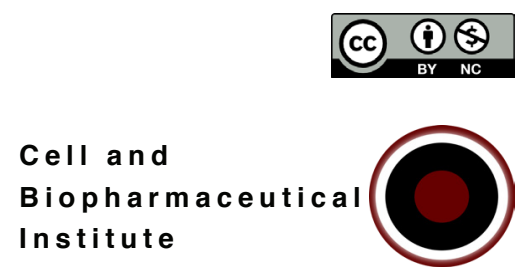


It is the main metabolite of artesunate and artemether, as well as an antimalarial compound on its own, which exhibits tautomerism of $\alpha$ and $\beta$ epimerization in solution and is chemically unstable in ambient temperature. ${ }^{3}$ DHA acts like amorphous powder with melting point of $164^{\circ} \mathrm{C}$, and with $\mathrm{pKa}$ 12.6, DHA is neutral at physiological $\mathrm{pH}$ and has moderate lipophilicity with four values of water solubility $(8.4 ; 50 ; 62 ; 168 \mathrm{mg} / \mathrm{L})$ and two values of octanol-water partition coefficient $(\log \mathrm{P})$ of 2.35 and 2.9. ${ }^{4}$

As artemisinin derivative, analytical methods to quantify plasma DHA have been studied since the earliest roll out of ACTs and some pitfalls have been identified. ${ }^{5-13}$ High performance liquid chromatography (HPLC) with ultraviolet (UV) detection was proven resilient for most drugs, however it was difficult to be applied to DHA since the compound lack of chromophore moieties. Several methods to yield UV-absorbing products were able to increase selectivity and sensitivity, but with a set of drawbacks which requires rigorously controlled anaerobic conditions and deoxygenation of the samples and mobile phases. The use of tandem mass spectrometry greatly improves selectivity and sensitivity.

The emergence of resistant Plasmodium falciparum to artemisinin in five neighboring countries, i.e., Cambodia, Thailand, Myanmar, Vietnam, and Lao PDR has generated concerns to the National Malaria Control Program (NMCP) as it would hamper malaria elimination program. ${ }^{14,15}$ Pharmacokinetic (PK) study or in essence, a drug-level study, is particularly of importance in order to distinguish treatment failures due to true resistance or inadequate drug concentrations. However, this kind of study is not commonly done in Indonesia due to limited laboratory capability. In line with the artemisinin resistance monitoring program, this study was conducted with the goal of building laboratory capacity at the National Institute of Health Research and Development (NIHRD), Ministry of Health of Indonesia. We investigated clinical applicability of a validated liquid chromatography/tandem mass spectrometry (LC-MS/MS) assay to quantify plasma concentration of DHA in healthy subjects from a single oral administration of fixed dose combination of Dihydroartemisinin-Piperaquine.

\section{Materials and methods}

This study was ethically approved by the Ethics Committee of Faculty of Medicine, University of Indonesia (No. 360/ UN2.F1/ETIK/2016). The selected reference assay was validated for the determination of DHA and artemether in human plasma and published in previous article. ${ }^{16}$

\section{Chemicals and Reagents}

DHA and stable isotope-labeled (SIL) internal standard (SIL-DHA) was acquired from the WorldWide Antimalarial Resistance Network (WWARN) for their reference material program. The structures as shown on the certificate of analysis are in Figure 1. Acetonitrile (HPLC-grade), methanol (pro analysis) and HPLC-water were obtained from Merck Chemicals and Life Sciences (Darmstadt, Germany). Ammonium acetate (LC-MS grade) was obtained from Sigma-Aldrich (Darmstadt, Germany). Ammonium acetate in HPLC-water was mixed to obtained ammonium acetate buffer solutions and the $\mathrm{pH}$ was adjusted with acetic acid from Merck Chemicals and Life Sciences (Darmstadt, Germany). Blank human plasma (common plasma with no DHA) was obtained from the Indonesian Red Cross (Jakarta, Indonesia) and citrate phosphate dextrose with adenine (CPDA-1) solution was used as anticoagulant.
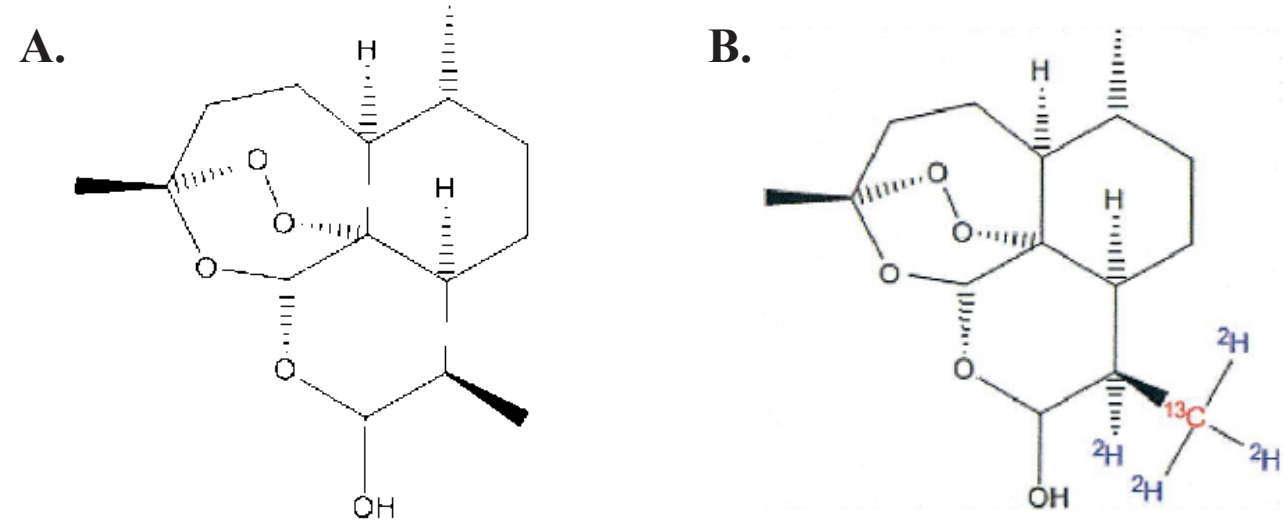

Figure 1. Structures of DHA (A) and SIL-DHA (B). 


\section{Instrumentations}

Waters Acquity UPLCTM H-Class system coupled with triple quadruple (TQD) mass spectrometry (Waters Corp., Milford, USA) was used for the validation experiments. The LC system consisted of a quaternary (binary pump with two channels in each pump) LC pump with vacuum degasser, an autosampler with flow-through needle, a column with active pre-heater properties. MassLynx ${ }^{\mathrm{TM}}$ Mass Spectrometry Software was used for data acquisition and quantification. The stationary phase was Waters Acquity UPLC ${ }^{\text {TM }}$ BEH C18 (50 × $2.1 \mathrm{~mm}, 1.7 \mu \mathrm{m}$, Waters Corp., Milford, USA) column. The mobile phase was consistent with the reference assay, which is a mixture of acetonitrile and ammonium acetate $10 \mathrm{mM} \mathrm{pH} 3.5(50: 50, \mathrm{v} / \mathrm{v})$. It was set at a flow rate of $0.3 \mathrm{~mL} / \mathrm{minute}$ in isocratic condition.

The MS conditions were optimized by directly injecting $1000 \mathrm{ng} / \mathrm{mL}$ standard solution of DHA mixed with $100 \mathrm{ng} / \mathrm{mL}$ SIL-DHA solution to the MS. Argon gas was set to 7 psi and nitrogen gas at $90-100$ psi. Electrospray ionization (ESI) was set in positive ionization mode. The MS console was set on autotune. Multiple reaction monitoring (MRM) was used for the quantification of DHA and SILDHA at transitions of $\mathrm{m} / \mathrm{z} 302$ to 163 and $\mathrm{m} / \mathrm{z} 307$ to 272 , respectively with dwell time 0.2 second. Electrospray ionization (ESI) was set in positive ionization mode. The cone voltage was $12 \mathrm{~V}$ for DHA and $14 \mathrm{~V}$ for SIL-DHA. The collision energy was $20 \mathrm{eV}$ for DHA and $12 \mathrm{eV}$ for SILDHA.

\section{Stock and Working Solutions, Calibration Standards and Quality Control Samples}

Stock solutions for DHA and SIL-DHA were prepared at concentration of $1,000 \mu \mathrm{g} / \mathrm{mL}$ in ethanol. Working solution of SIL-DHA was prepared by dilution of stock solution to achieve concentration of $100 \mu \mathrm{g} / \mathrm{mL}$ in ethanol-water (50$50, \mathrm{v} / \mathrm{v})$. Working solutions of DHA ranging from $100 \mathrm{ng} /$ $\mathrm{mL}$ to $100 \mu \mathrm{g} / \mathrm{mL}$ were also prepared by serial dilution of stock solution in ethanol-water (50-50, v/v). These solutions were kept on ice during use. On the day of assay, a fix concentration of SIL-DHA at $100 \mathrm{ng} / \mathrm{mL}$ was obtained by diluting working solution of SIL-DHA with plasma-water (50-50, v/v containing sodium fluoride/potassium oxalate $2 / 3 \mathrm{mg} / \mathrm{mL}$ ).

A ten-point calibration curve was prepared for DHA by spiking blank plasma to yield calibration concentration of 1 , $3,5,10,25,50,100,250,500$, and $1,000 \mathrm{ng} / \mathrm{mL}$, in addition to the blank and zero plasma. Quality control (QC) samples for DHA (low, medium, high) were prepared similarly for concentrations of 5, 402 and $800 \mathrm{ng} / \mathrm{mL}$. The QC low (QCL) concentration was 5 times the LLOQ, QC high $(\mathrm{QCH})$ concentration was $80 \%$ of the highest concentration of the standard solution. The QC medium (QCM) concentration was obtained by adding QC low and high concentrations divided by two. These solutions were kept at the minimum temperature of $4^{\circ} \mathrm{C}$ until day of the assay.

\section{Sample Preparation}

Plasma samples were prepared according to the reference assay using solid-phase extraction (SPE) method in $\mu$-elution Oasis ${ }^{\circledR}$ HLB 96-wellplate (Waters, USA) with slight modification to the plasma volume. One hundred and fifty microliters of internal standard solution $(100 \mathrm{ng} / \mathrm{mL}$ SIL-DHA in plasma-water $(50: 50, \mathrm{v} / \mathrm{v})$ containing sodium fluoride/potassium oxalate $2 / 3 \mathrm{mg} / \mathrm{mL}$ ) was added to 100 $\mu \mathrm{L}$ plasma in polypropylene tube on ice. Acetonitrile (750 $\mu \mathrm{L})$, followed by methanol $(750 \mu \mathrm{L})$ and water $(200 \mu \mathrm{L})$ were loaded to activate and conditioned the SPE wells. The samples $(250 \mu \mathrm{L}$ each) were pipetted onto the activated wells and drawn through with low pressure vacuum. Then, water $(300 \mu \mathrm{L})$ was loaded to wash the wells, drawn through with medium pressure vacuum.

The pressure was increased briefly to full vacuum to make sure all liquids were drawn through. The SPE column tips were dried with tissue paper before inserting a 96-collection plate $(0.5 \mathrm{~mL})$ into the vacuum manifold. Methanol-acetonitrile $(100 \mu \mathrm{L}, 90: 10 \mathrm{v} / \mathrm{v})$ followed by water $(100 \mu \mathrm{L})$ were used to elute the wells with low pressure vacuum. The eluates were briefly mixed before refrigeration at $4^{\circ} \mathrm{C}$ for 15 hours. As stated on the reference assay, this step was needed to enable $\alpha / \beta$-DHA epimer equilibration. Five $\mu \mathrm{L}$ of eluates was injected into the LC-MS/MS system.

\section{Assay Validations}

Partial validation experiments were performed according to the European Medicines Agency (EMA) guideline. ${ }^{17}$ The method was tested against several validation parameters, which include linearity, lower limit of quantitation (LLOQ), selectivity, accuracy, precision, matrix effects, and stability. Linearity was evaluated by preparing calibration standards of the previously mentioned concentrations in four replicates. Peak area ratios of the analyte to the IS versus the nominal concentrations in quadruplet were plotted to obtained calibration standards over the range of 1-1,000 ng/ $\mathrm{mL}$ concentrations. The calibration curve with $1 / \mathrm{x}^{2}$ weighed 
linear regression was chosen for quantification, since it was expected that the residual errors will not be randomly distributed. It is generally acceptable when the correlation coefficient (r) of the calibration curves was greater than 0.99 and the bias of the calculated concentrations was within $\pm 15 \%$ of the nominal concentrations, except at the LLOQ concentration of which the deviation can be within $\pm 20 \%$.

LLOQ was established by analyzing six blank plasma samples, first by spiking it with the LLOQ concentration of the reference assay and analyzing the response. The analyte response should be reliably precise and accurate (acceptable if each criterion is less than $20 \%$ ). When the analyte response was within acceptable range, the concentration was lowered by half and the response was analyzed once again.

Selectivity was evaluated by analyzing six blank plasma samples in comparison to spiked plasma at LLOQ concentration. The response of endogenous or other components in the plasma should be less than $20 \%$ of the LLOQ for the analyte and 5\% for the IS.

Intra-assay accuracy and precision experiments were determined by repeating the analysis of each QC standard five times. This was performed at least in three different batches (inter-assay) and the deviation within $\pm 15 \%$ of the true value was acceptable, except at the lower LLOQ concentration of which the deviation within $\pm 20 \%$ was acceptable.

Matrix effect of analyte and IS was evaluated in six lots of blank plasma from different donors, extracted and spiked at QCL, QCM and QCH concentrations, in addition to pure standard solutions in equivalent concentrations. Peak area in post-extraction samples spiked with analyte or IS were compared with peak areas of standard solutions in equivalent concentrations to obtain matrix factor for each analyte and IS (matrix factor $(\mathrm{MF})=$ peak response in presence of matrix ions / peak response in absence of matrix ions). IS-normalized MF were calculated by dividing the MF of analyte to the MF of IS. Variation of IS-normalized MF from six lots of plasma should not exceed 15\%.

Stability was evaluated with blank matrix spiked with QCL and QCH concentrations in triplicates right after the preparation, at 6 hours and 12 hours after refrigeration in $4^{\circ} \mathrm{C}$. The mean concentration should be within $\pm 15 \%$ of the nominal concentration.

\section{Plasma DHA Sample Collection from Healthy Adults}

Five healthy male subjects between 18 and 40 years of age with body mass index (BMI) range between 18.5 and
$24.9 \mathrm{~kg} / \mathrm{m}^{2}$, normal vital signs, normal standard physical examination, normal values of routine hematology, blood glucose, liver and renal function tests, and normal electrocardiogram (ECG) readings were consented and recruited in this study. They did not have any history of having allergic/hypersensitivity reaction to either DHA or PIP, active psychiatric condition, or alcohol or drug dependence, and did not take any concomitant medications or under long-term treatment.

The study drug was a fixed-dose oral formulation (tablet) containing $40 \mathrm{mg}$ DHA and $320 \mathrm{mg}$ PIP phosphate obtained from the Indonesian NMCP. Drug dosage was individually adjusted by body weight (DHA $2-4 \mathrm{mg} / \mathrm{kg}$ body weight/day and PIP $16-32 \mathrm{mg} / \mathrm{kg}$ body weight/day) and rounded up to the nearest quarter. The drug dose was administered once with water under the supervision of study physician at least 3 hours after their last meal. The participants continued to fast up to 3 hours after the dose and monitored for adverse events. No specific diet restriction was applied to the participants.

Venous blood was collected just before drug administration (time 0 ) and at $0.25,0.5,0.75,1,1.25$, $1.5,2,3,4,6$, and 8 hours after drug administration in ethylenediaminetetraacetic acid (EDTA) tubes. Within 30 minutes after collection, the blood samples were centrifuged for 15 minutes at $3000 \mathrm{rpm}$. The resulting plasma were aliquoted, labelled and stored at $-70^{\circ} \mathrm{C}$ or lower prior to analysis.

The PK parameters were modelled using noncompartment analysis (NCA) using Stata ${ }^{\circledR}$ software version 14 (StataCorp, Texas, USA). Peak drug concentration $\left(C_{\max }\right)$ and the actual time when the peak concentration was measured $\left(t_{\max }\right)$ was observed directly from the plotted mean plasma drug concentrations versus time. Other parameters were calculated from the logarithmic plot of mean plasma concentrations versus time, such as the elimination rate constant and half-life $\left(t_{1 / 2}\right)$, volume of distribution $\left(V_{d}\right)$ and clearance $(\mathrm{CL})$. The areas under the curve (AUC) from time 0 to $t$ were calculated using loglinear trapezoidal rule.

\section{Results}

\section{Sample Preparation}

Several experiments $(n=3)$ of sample preparation using 50 $\mu \mathrm{L}$ plasma as stated in the reference assay failed to produce sufficient peak when injected to the LC-MS/MS system. 
Doubling the volume of plasma to $100 \mu \mathrm{L}$ was able to correct the peak and subsequently chosen to be used in this study.

\section{Mass Spectra Analysis and Chromatographic Separation}

The optimized chromatographic condition was achieved by autotune. Full scan mass spectra were acquired in positive ion mode. The cone voltage was $12 \mathrm{~V}$ for DHA and $14 \mathrm{~V}$ for SIL-DHA. The collision energy was $20 \mathrm{~V}$ for DHA and $12 \mathrm{~V}$ for SIL-DHA. In the direct infusion experiment, the mass spectra for DHA and SIL-DHA revealed peaks at mass to charge ratio $(\mathrm{m} / \mathrm{z}) 302$ and 307, respectively. The most stable abundant fragment ion observed product MS/MS spectrum was at m/z 163 for DHA and 272 for SIL-DHA. Figure 2 showed the collision-induced dissociation (CID) mass spectra for DHA (mass transition $302 \rightarrow 163 \mathrm{~m} / \mathrm{z}$ ) and SIL-DHA (mass transition $307 \rightarrow 272 \mathrm{~m} / \mathrm{z}$ ).

The total ion chromatogram for standard solution of DHA is shown in Figure 3 using mobile phase of acetonitrile and ammonium acetate $10 \mathrm{mM}$ pH 3.5 (50:50, $\mathrm{v} / \mathrm{v}$ ) at flow rate $0.3 \mathrm{~mL} / \mathrm{min}$. Since the separation of $\alpha$ and $\beta$-DHA was not clearly defined, both peaks were used for the quantification of DHA. Run time was less than 10 minutes.

\section{Assay Validations: Linearity, Lower Limit of Quantification and Selectivity}

Linearity of the assay was observed on the calibration curve at the concentration range of $1-1,000 \mathrm{ng} / \mathrm{mL}$ with correlation coefficient $(\mathrm{r} 2)>0.99$. The mean accuracy and precision at each concentration of the standard in the calibration curve are shown in Table 1.

The LLOQ for DHA was $1 \mathrm{ng} / \mathrm{mL}$ with coefficient of variation $(\% \mathrm{CV})$ and bias (standard deviation $/ \mathrm{SD})$ of back calculated of each concentration were $4.83 \%$ and 0.05 , respectively. All blank plasma sources produced signals that contributed lower than $20 \%$ of a standard at LLOQ and less than $5 \%$ for IS.
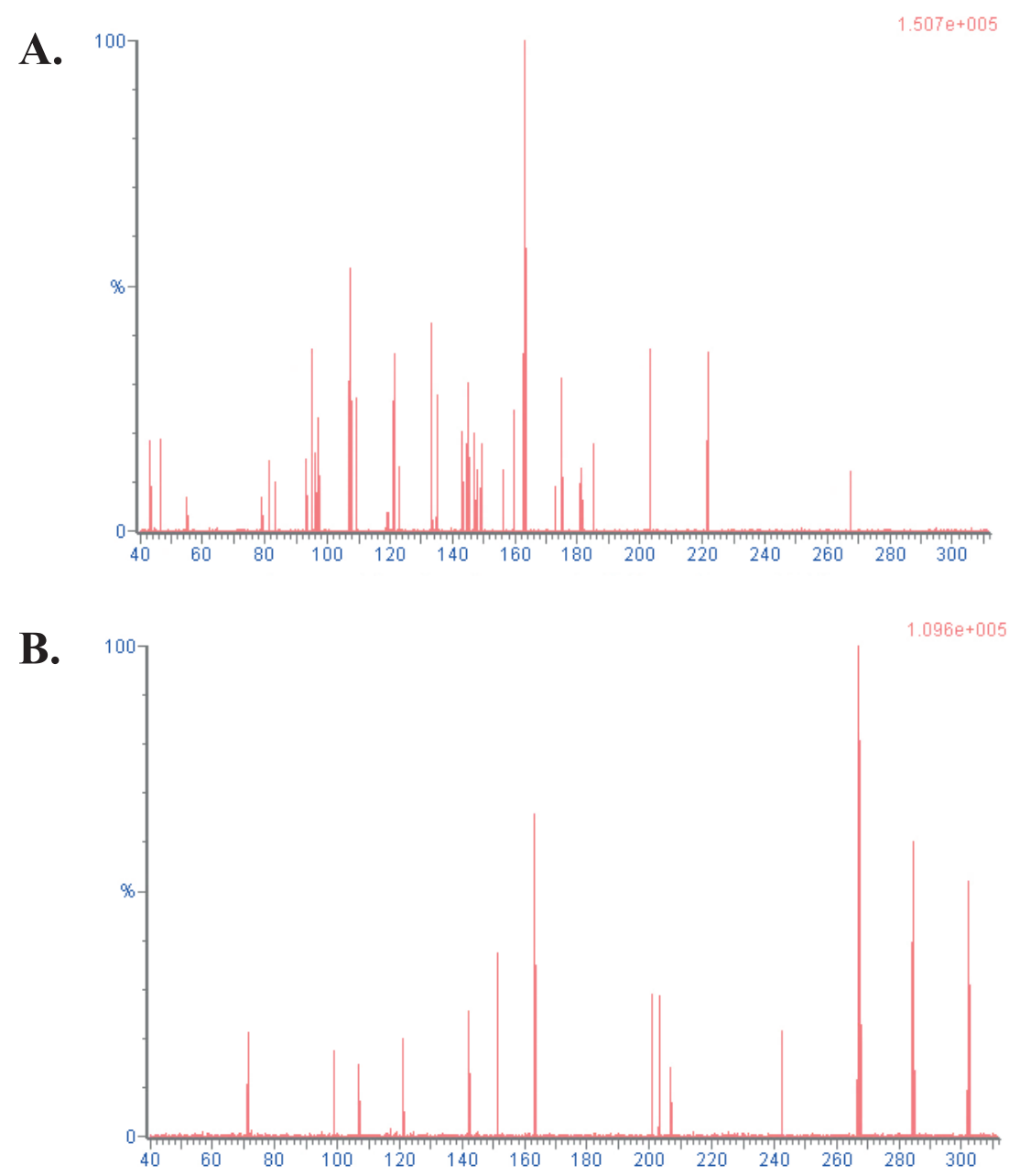

Figure 2. Product ion spectra (positive ESI, $\mathrm{m} / \mathrm{z}$ 40-350) of DHA (302/163) (A) and SIL-DHA (307/272) (B). 


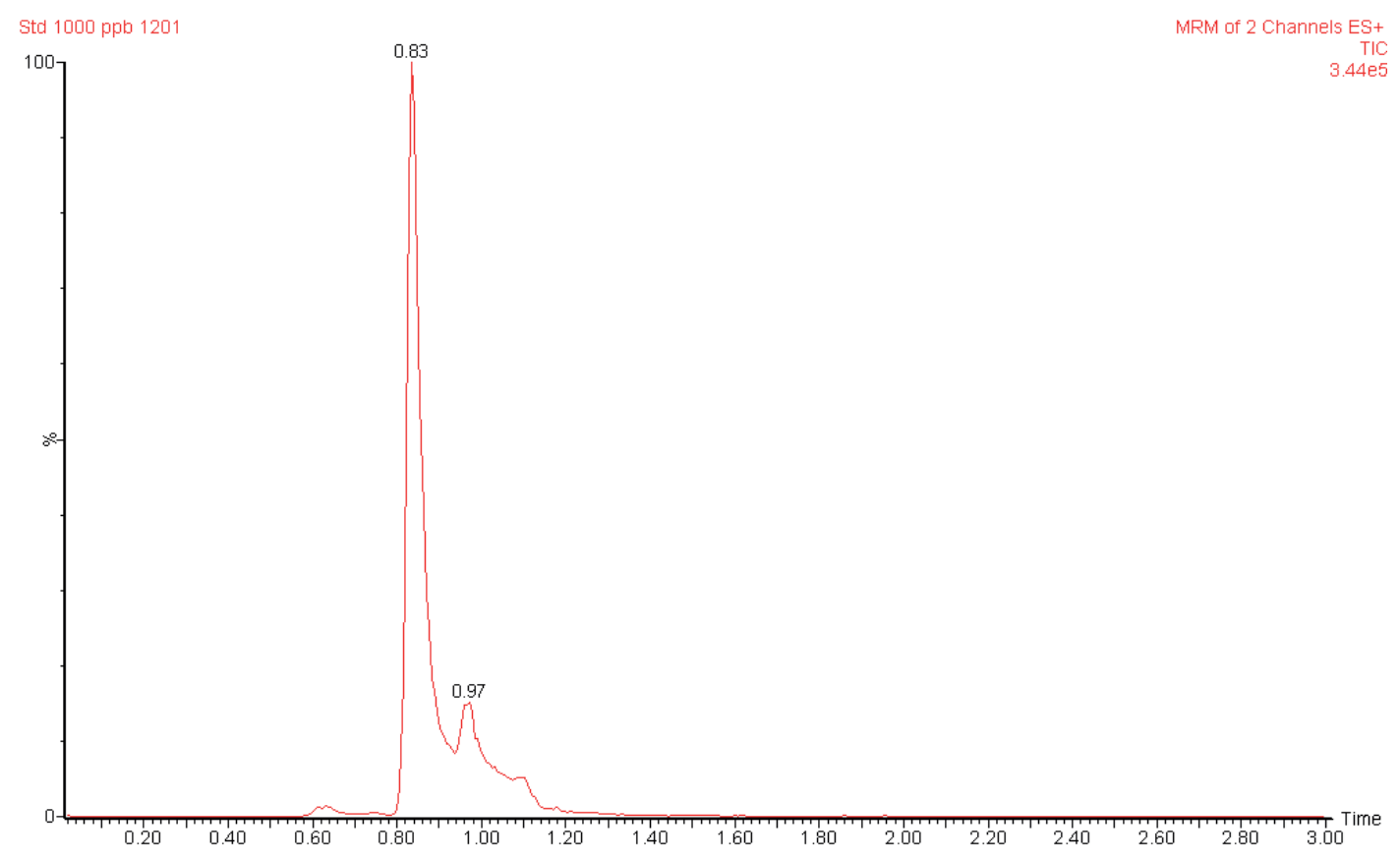

Figure 3. The total ion chromatogram of dihydroartemisinin from standard solution on the Waters Acquity UPLCTM BEH C18 column $(50 \times 2.1 \mathrm{~mm}, 1.7 \mu \mathrm{m})$ at flow rate $0.3 \mathrm{~mL} / \mathrm{min}$ with mobile phase containing a mixture of acetonitrile and ammonium acetate $10 \mathrm{mM}$ pH 3.5 (50:50, v/v).

\section{Accuracy and Precision}

The results of intra- and inter-day precision and accuracy of the assay method are summarized in Table 2. Intraand inter-day accuracies were 99.99-102.85\% and 96.45$107.47 \%$, respectively. Intra- and inter-day precisions (relative standard deviation /RSD) were 2.79-4.16\% and $1.46-3.04 \%$, respectively. Results show that the accuracy and precision values were within the acceptable criteria.

\section{Matrix Effects}

The normalized matrix effect of DHA to IS were close to 1 with acceptable variations (below 15\%), summarized in Table 3. These data showed that under the current conditions, the event of ion suppression or enhancement (matrix effect) from human plasma can be ignored.

\section{Stability}

Poor stability of DHA at ambient temperature has been demonstrated. According to the reference method, working on ice throughout the sample preparation is required. The mean recovery $(n=5)$ after 24 hours refrigeration at $4^{\circ} \mathrm{C}$ at low concentration was $4.83 \mathrm{ng} / \mathrm{mL}(3.44 \% \mathrm{CV})$ and 781.34 $\mathrm{ng} / \mathrm{mL}$ at high concentration $(2.19 \% \mathrm{CV})$. The stability test results of DHA in human plasma samples are summarized in Table 4. Results indicate adequate stability of DHA enduring sample preparation process and storage conditions.

Application of the Assay to Plasma Samples of DHA from Healthy Subjects

To describe clinical application of the assay, the pharmacokinetics of DHA following single oral

Table 1. Back-calculated concentrations of standard curve for DHA in human plasma.

\begin{tabular}{|c|c|c|c|c|c|c|c|c|c|c|}
\hline \multirow{2}{*}{ DHA } & \multicolumn{10}{|c|}{ Nominal Concentration (ng/mL) } \\
\hline & 1 & 3 & 5 & 10 & 25 & 50 & 100 & 250 & 500 & 1000 \\
\hline Average $(n=4)$ & 0.97 & 2.93 & 4.75 & 9.26 & 23.68 & 47.67 & 99.83 & 249.38 & 510.43 & 995.12 \\
\hline SD & 0.03 & 0.21 & 0.12 & 0.14 & 1.16 & 0.8 & 1.1 & 8.92 & 3.25 & 24.49 \\
\hline $\mathrm{CV}(\%)$ & 3.27 & 7.19 & 2.49 & 1.46 & 4.88 & 1.67 & 1.11 & 3.58 & 0.64 & 2.46 \\
\hline Accuracy (\%) & 96.67 & 97.61 & 94.92 & 92.64 & 94.72 & 95.34 & 99.83 & 99.75 & 102.09 & 99.51 \\
\hline
\end{tabular}


Table 2. Intra- and inter-day accuracy and precision of DHA in human plasma.

\begin{tabular}{lccccc}
\hline \multirow{2}{*}{ DHA } & \multicolumn{2}{c}{ Inter-day } & & \multicolumn{2}{c}{ Intra-day } \\
\cline { 2 - 3 } \cline { 5 - 6 } & $\begin{array}{c}\text { Precision } \\
\text { (\%RSD) }\end{array}$ & $\begin{array}{c}\text { Accuracy } \\
(\text { \%) }\end{array}$ & & $\begin{array}{c}\text { Precision } \\
\text { (\%RSD) }\end{array}$ & $\begin{array}{c}\text { Accuracy } \\
(\%)\end{array}$ \\
\hline $\mathrm{LLOQ}, 1 \mathrm{ng} / \mathrm{mL}$ & 4.16 & 102.85 & & 2.4 & 104.11 \\
$\mathrm{QCL}, 5 \mathrm{ng} / \mathrm{mL}$ & 3.67 & 101.43 & & 3.04 & 96.45 \\
$\mathrm{QCM}, 402 \mathrm{ng} / \mathrm{mL}$ & 3.16 & 99.99 & & 1.46 & 107.47 \\
$\mathrm{QCH}, 800 \mathrm{ng} / \mathrm{mL}$ & 2.79 & 100.53 & 2.66 & 99.45 \\
\hline
\end{tabular}

administration of fixed-dose combination of $40 \mathrm{mg}$ DHA and $320 \mathrm{mg}$ PIP in 5 healthy male adults was characterized. The median age was 23 years (range 18-39) and median body mass index was $23.25 \mathrm{~kg} / \mathrm{m}^{2}$ (range 18.75-24.63). The average dose of DHA was given at $2.3 \mathrm{mg} / \mathrm{kg}$ body weight.

The mean plasma concentration-time profiles of DHA in 5 healthy subjects is shown in Figure 4. A $C_{\max }$ of 300.4213 $\mathrm{ng} / \mathrm{mL}$ was reached at $1.5 \mathrm{~h}\left(t_{\max }\right)$ after drug administration. The elimination rate constant was $0.85 / \mathrm{h}$ and the $\mathrm{t}_{1 / 2}$ was 0.81 hour. The $\mathrm{AUC}_{0 \text {-last }}$ of the mean plasma concentration-time curve of DHA was $611.79 \mathrm{ng} / \mathrm{mL}$ and $\mathrm{AUC}_{0-\infty}$ was 613.680 $\mathrm{ng} / \mathrm{mL}$. The $\mathrm{CL}$ was $3.75 \mathrm{~L} / \mathrm{h} / \mathrm{kg}$ and the apparent volume of distribution $\left(V_{d}\right)$ was $4.36 \mathrm{~L} / \mathrm{kg}$.

\section{Discussion}

This study aimed to established laboratory readiness for pharmacokinetic or drug level study, therefore we used an already-validated assay as the reference and did not develop novel methods for the quantification of DHA for practical reasons. The reference assay was replicated to the extent possible, but some modifications were inevitable due to the differences in the LC-MS/MS detection system and anticoagulants used in the reference assay, plasma and clinical samples. Partial validation was required according to the EMA guideline, but the specific parameters were not rigidly defined. As such, they can range from within-run precision accuracy evaluation to near full validation.

In this study, the following parameters were evaluated, i.e., linearity, LLOQ, selectivity, accuracy, precision, stability and matrix effects. Carry over and the effect of different anticoagulant to plasma and clinical samples were later deemed necessary, but not done in this study due to shortage of materials and reagents. On the other hand, stability was an optional parameter that was not required but done in this study. Our first lesson learned from this study was to better-plan the assay validation experiments. The parameters for partial validations should be carefully selected based on risk-based approach, considering the impact of the modifications to the method and whether the changes considered significant. ${ }^{18}$

This study used plasma samples from the Indonesian Red Cross and citrate phosphate dextrose (CPDA-1) solution was used as anticoagulant, whereas the reference assay was validated in fluoro-oxalate plasma and blood samples from healthy adults were collected in ethylenediamine tetra-

Table 3. Matrix effects DHA and SIL-DHA spiked in extracted blank human plasma vs. spiked in elution solution.

\begin{tabular}{lccccccccc}
\hline & Blank A & Blank B & Blank C & Blank D & Blank E & Blank F & Average & SD & \%CV \\
\hline DHA, 5 ng/mL & 3.061 & 2.463 & 2.296 & 2.458 & 2.512 & 2.086 & 2.479 & 0.3 & 13.1 \\
SIL-DHA, 100 ng/mL & 3.169 & 2.436 & 2.377 & 2.487 & 2.506 & 2.131 & 2.518 & 0.3 & 13.8 \\
Normalized response & 0.966 & 1.011 & 0.966 & 0.988 & 1.002 & 0.979 & 0.986 & 0 & 1.9 \\
\hline DHA, $402 \mathrm{ng} / \mathrm{mL}$ & 3.85 & 3.19 & 2.96 & 3.07 & 3.32 & 3.81 & 3.367 & 0.4 & 11.2 \\
SIL-DHA, 100 ng/mL & 2.601 & 2.246 & 1.973 & 2.06 & 2.228 & 2.683 & 2.299 & 0.3 & 12.5 \\
Normalized response & 1.48 & 1.42 & 1.5 & 1.49 & 1.49 & 1.42 & 1.467 & 0 & 2.5 \\
\hline DHA, 800 ng/mL & 2.307 & 2.049 & 1.699 & 1.866 & 1.744 & 1.694 & 1.893 & 0.2 & 12.9 \\
SIL-DHA, 100 ng/mL & 2.271 & 2.033 & 1.683 & 1.856 & 1.681 & 1.759 & 1.88 & 0.2 & 12.3 \\
Normalized response & 1.016 & 1.008 & 1.009 & 1.006 & 1.037 & 0.963 & 1.007 & 0 & 2.4 \\
\hline
\end{tabular}


Table 4. Stability of DHA in human plasma samples in temperature of $4^{\circ} \mathrm{C}$.

\begin{tabular}{llccc}
\hline & \multicolumn{1}{c}{ DHA } & Fresh & $\mathbf{6 ~ h}$ & $\mathbf{2 4} \mathbf{~ h}$ \\
\hline $\mathbf{5} \mathbf{~ n g} / \mathbf{m L}$ & Average & 5.26 & 4.96 & 4.83 \\
& SD & 0.22 & 0.25 & 0.17 \\
& CV $(\%)$ & 4.2 & 4.96 & 3.44 \\
& Accuracy & 105.26 & 99.27 & 96.54 \\
\hline $\mathbf{8 0 0} \mathbf{~ n g / m L}$ & Average & 807.51 & 808.89 & 781.34 \\
& SD & 18.6 & 19.26 & 17.08 \\
& CV $(\%)$ & 2.3 & 2.38 & 2.19 \\
& Accuracy & 100.94 & 101.11 & 97.67 \\
\hline
\end{tabular}

acetic acid (EDTA) tubes. The EMA Guideline stated that partial validation is required when there were changes in anticoagulants. To our knowledge, this area has not been widely investigated since only one study investigated DHA concentration in fluoro-oxalate plasma to heparin plasma. ${ }^{19}$ A separate validation experiments to address this issue would have been a valuable insight, thus our second lesson learned from this study.

This study's DHA chromatogram showed peak asymmetry in the form of marginal peak tailing. Peak asymmetry is a common sign of LC separation problem and several possible causes were identified. The first was the difference in the stationary phase. The reference assay used Hypersil Gold C18 $(100 \times 2.1 \mathrm{~mm}, 5 \mu \mathrm{m})$, while this study used Waters Acquity UPLC ${ }^{\text {TM }}$ BEH C18 column (50 $\times 2.1 \mathrm{~mm}, 1.7 \mu \mathrm{m})$ column. Both columns have different intrinsic characteristics, such as relative hydrophobicity, relative polarity, efficiency, etc., that may have influenced chromatographic separation. ${ }^{20}$

The mobile phase of acetonitrile and ammonium acetate $10 \mathrm{mM}$ pH $3.5(50: 50, \mathrm{v} / \mathrm{v})$ and injection volume of $5 \mu \mathrm{L}$ was directly transferred from the reference assay without further adjustments to the composition of the mobile phase (ratio of aqueous to organic solvent) to correct the peak. This was identified as the second cause for peak asymmetry in this study. In addition, the reference assay used gradient condition that can further minimized tailing, while this study used isocratic condition, this was the third cause for peak asymmetry in this study.

Low sensitivity of the MS detection to DHA in this study was observed with the doubling of plasma volume to $100 \mu \mathrm{L}$. The reference assay used API 5000 triple quadrupole mass spectrometer (Applied Biosystems/MDS SCIEX, Foster City, USA), while this study used Waters

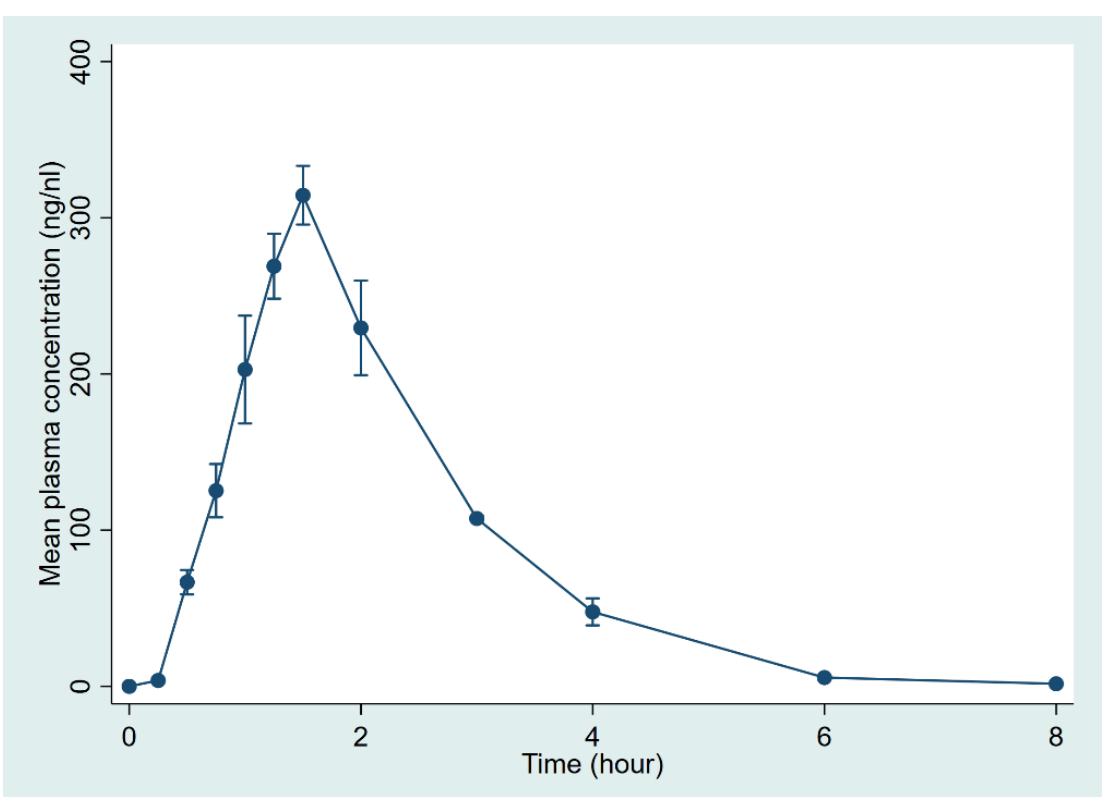

Figure 4. Mean plasma concentrationtime profiles of dihydroartemisinin in five healthy male adults after single oral administration of fixed dose combination of DihydroartemisininPiperaquine. 
TQ Mass Spectrometers (Waters Corp., Milford, USA). During optimization, the MS condition in this study was achieved by autotune program, which means the MS tuning parameter was set according to factory setting, except for the cone voltage and collision energy. No adjustments were made to the temperature and gas settings that could have improved ionization. The Sciex API 5000 used by the reference assay might also be more sensitive than Waters TQ Mass Spectrometers, even though the latter was more user friendly for both their software and hardware.

The assay performance for DHA was incompletely validated against several parameters such as linearity, lower limit of quantitation (LLOQ), selectivity, accuracy, precision, stability and matrix effects; while leaving out other required parameters such as carry over and anticoagulants. Calibration curve was shown to be linear with a correlation coefficient greater than 0.995 over a concentration range of $1-1,000 \mathrm{ng} / \mathrm{mL}$. Bias and variation for accuracy and precision were in the range of $15 \%(20 \%$ at the lower limit of quantification). Matrix effect from human plasma was negligible (less than 15\%).

Clinical applicability was demonstrated when analyzing plasma drug concentrations after oral administration of fixed drug combination of DHA-PIP in 5 healthy subjects. Several studies addressing the PK of DHA using NCA following oral fixed dose formulation of DHAPIP in healthy young adults in Asian countries showed fairly similar results with this study. ${ }^{21-23}$ The Cmax was between 300 and $387 \mathrm{ng} / \mathrm{ml}$ and tmax was achieved within 1-1.5 hours after drug administration. The half-life was also consistent with other studies, between 0.98 and 2.03 hour. However, there appeared to be variability in the apparent volume of distribution of DHA i.e., 3.37; 4.38; 5.53 and $6.31 \mathrm{~L} / \mathrm{kg}$. The same variability was noted in the clearance of DHA i.e., $1.32 ; 2.21 ; 3.75$ and $4.54 \mathrm{~L} / \mathrm{h} / \mathrm{kg}$ that could have been caused by the differences in the subjects's body weight, but overall DHA was cleared after 8 hours.

\section{Conclusion}

Validation experiments to the LC-MS/MS method for the quantification of plasma DHA was successful for selectivity, linearity, lower limit of quantitation, accuracy, precision, matrix effect and stability parameters. Other validation parameters such as carry over and the role of anticoagulants were not tested in this study. Although clinical applicability was demonstrated, this method needed to be improved with additional measures to address the not-tested validation parameters. Additional evaluation to artesunate might be good to explore, since this could be done with the samealready-in-placed LC condition as DHA.

\section{Acknowledgements}

This study was supported by the National Institute of Health Research and Development, Ministry of Health of Indonesia through the Indonesia Research Partnership on Infectious Diseases (INA-RESPOND) and the Chulabhorn International College of Medicine, Thammasat University, Center of Excellence in Pharmacology and Malaria and Cholangiocarcinoma of Thammasat University.

\section{References}

1. World Health Organization (WHO). World Malaria Report 2018. Geneva: World Health Organization; 2018.

2. Balint GA. Artemisinin and its derivatives: an important new class of antimalarial agents. Pharmacol Ther. 2001; 90(2-3): 261-5.

3. Parapini S, Olliaro P, Navaratnam V, Taramelli D, Basilico N. Stability of the antimalarial drug dihydroartemisinin under physiologically relevant conditions: implications for clinical treatment and pharmacokinetic and in vitro assays. Antimicrob Agents Chemother. 2015; 59(7): 4046-52.

4. Ali S, Jafery N, Farhat K, Waheed A. Analysis of Artemether and Dihydroartemisinin by high performance high liquid chromatography in biological fluids-issues and solutions. Pak J Pharm Sci. 2017; 30(4): 1395-401.

5. Karbwang J, Na-Bangchang K, Molunto P, Banmairuroi V, Congpuong K. Determination of artemether and its major metabolite, dihydroartemisinin, in plasma using high-performance liquid chromatography with electrochemical detection. J Chromatogr B. 1997; 690(1-2): 259-65.

6. Navaratnam V, Mordi MN, Mansor SM. Simultaneous determination of artesunic acid and dihydroartemisinin in blood plasma by highperformance liquid chromatography for application in clinical pharmacological studies. J Chromatogr B. 1997; 692(1): 157-62.

7. Souppart C, Gauducheau N, Sandrenan N, Richard F. Development and validation of a high-performance liquid chromatography-mass spectrometry assay for the determination of artemether and its metabolite dihydroartemisinin in human plasma. J Chromatogr B. 2002; 774(2): 195-203.

8. Sabarinath S, Rajanikanth M, Madhusudanan KP, Gupta RC. A sensitive and selective liquid chromatographic/ electrospray ionization tandem mass spectrometric assay for the simultaneous quantification of a-,b-arteether and its metabolite dihydroartemisinin in plasma, useful for pharmacokinetic studies. J Mass Spectrom. 2003; 38: 732-42.

9. Naik H, Murry DJ, Kirsch LE, Fleckenstein L. Development and validation of a high-performance liquid chromatographymass spectroscopy assay for determination of artesunate and dihydroartemisinin in human plasma. J Chromatogr B. 2005; 816(233-42): 233-42. 
10. Peys E, Vandenkerckhove J, Hemel JV, Sas B. Simultaneous determination of b-artemether and its metabolite dihydroartemisininin human plasma and urine by a highperformance liquid chromatography-mass spectrometry assay using electrospray ionisation. Chromatographia. 2005; 61: 637-41.

11. Shi B, Yu Y, Li Z, Zhang L, Zhong Y, Su S, et al. Quantitative analysis of artemether and its metabolite dihydroartemisinin in human plasma by LC with tandem mass spectrometry. Chromatographia. 2006; 64: 523-30

12. Lai CS, Naira NK, Mansor SM, Olliaro PL, Navaratnam V. An analytical method with a single extraction procedure and two separate high performance liquid chromatographic systems for the determination of artesunate, dihydroartemisinin and mefloquine in human plasma for application in clinical pharmacological studies of the drug combination. J Chromatogr B. 2007; 857(2): 308-14.

13. Gu Y, Li Q, Melendez V, Weina P. Comparison of HPLC with electrochemical detection and LC-MS/MS for the separation and validation of artesunate and dihydroartemisinin in animal and human plasma. J Chromatogr B. 2008; 867(2): 213-18.

14. Sitohang V, Sariwati E, Fajariyani SB, Hwang D, Kurnia B, Hapsari RK, et al. Malaria elimination in Indonesia: halfway there. Lancet Glob Health. 2018; 6(6): e604-e6.

15. Ménard D, Khim N, Beghain J, Adegnika AA, Shafiul-Alam M, Amodu O, et al. A worldwide map of Plasmodium falciparum K13propeller polymorphisms. N Eng J Med. 2016; 374(25): 2453-64.

16. Hanpithakpong W, Kamanikom B, Dondorp A, Singhasivanon $\mathrm{P}$, White $\mathrm{N}$, Day $\mathrm{N}$, et al. A liquid chromatographic-tandem mass spectrometric method for determination of artesunate and itsmetabolite dihydroartemisinin in human plasma. J Chromatogr B. 2008; 876(1): 61-8.
17. EMA. Guideline on Bioanalytical Method Validation. London: European Medicines Agency; 2011.

18. Briggs RJ, Nicholson R, Vazvaei F, Busch J, Mabuchi M, Mahesh $\mathrm{KS}$, et al. Method transfer, partial validation, and cross validation: recommendations for best practices and harmonization from the global bioanalysis consortium harmonization team. AAPS J. 2014; 16(6): 1143-8.

19. Lindegardh N, Hanpithakpong W, Kamanikom B, Singhasivanon $\mathrm{P}$, Socheat D, Yi P, et al. Major pitfalls in the measurement of artemisinin derivatives in plasma in clinical studies. J Chromatogr B. 2008 ; 876(1): 54-60.

20. MAC MOD Analytical. Comparison Guide to C18 Reversed Phase HPLC Columns: Comparison Data on Commonly used C18 Phases. Pennsylvania: MAC MOD; 2008.

21. Hanboonkunupakarn B, van der Pluijm RW, Hoglund R, Pukrittayakamee S, Winterberg M, Mukaka M, et al. Sequential open label study of the safety, tolerability and pharmacokinetic interactions between dihydroartemisinin-piperaquin and mefloquine in healthy Thai adults. Antimicrob Agents Chemother. 2019; 63: e00060-19.

22. Hanboonkunupakarn B, Ashley EA, Jittamala P, Tarning J, Pukrittayakamee S, Hanpithakpong W, et al. Open-label crossover study of primaquine and dihydroartemisinin-piperaquine pharmacokinetics in healthy adult thai subjects. Antimicrob Agents Chemother. 2014; 58(12): 7340-6.

23. Chinh NT, Quang NN, Thanh NX, Dai B, Geue JP, Addison RS, et al. Pharmacokinetics and bioequivalence evaluation of two fixeddose tablet formulations of dihydroartemisinin and piperaquine in Vietnamese subjects. Antimicrob Agents Chemother. 2009; 53(2): 828-31. 